\title{
УГЛОВЫЕ ОПТИЧЕСКИЕ ХАРАКТЕРИСТИКИ ИЗЛУЧЕНИЯ, РАССЕЯННОГО ДВУХСЛОЙНЫМИ НАНОЧАСТИЦАМИ СИСТЕМЫ МЕТАЛЛ-ОКИСЕЛ
}

\author{
(C) 2016 г. Л. Г. Астафьева*, В. К. Пустовалов** \\ * Институт физики имени Б.И. Степанова НАН Беларуси, 220072 Минск, Беларусь \\ **Белорусский национальный технический университет, 220013 Минск, Беларусь \\ E-mail: astafev@dragon.bas-net.by \\ Поступила в редакцию 18.12.2015 г. \\ В окончательной редакщии 01.03.2016 г.
}

\begin{abstract}
Численно рассчитаны индикатрисы рассеяния излучения с длинами волн $\lambda=300,560$ и 1000 нм однородными наночастицами с радиусами $r_{0}=50,75,100$ нм и двухслойными наночастицами с радиусами ядра $r_{0}=40,65,90$ нм и толщиной оболочки $\Delta r=10$ нм. В качестве материалов однородных наночастиц использованы металлы $\mathrm{Ti}, \mathrm{Ni}, \mathrm{Zn}$ и окислы этих металлов $\mathrm{TiO}_{2}, \mathrm{NiO}, \mathrm{ZnO}$. B качестве материалов ядра и оболочек двухслойных наночастиц использованы металлы Ti, Ni, Zn и окислы этих металлов $\mathrm{TiO}_{2}, \mathrm{NiO}, \mathrm{ZnO}$ соответственно. Установлено, что наличие окисной оболочки на металлическом ядре из титана и никеля по сравнению с чисто металлической или чисто окисной диэлектрической наночастицами приводит к сушественному уменьшению обратного рассеяния для $\lambda=300$ нм.
\end{abstract}

DOI: $10.7868 / \mathrm{S} 0030403416070047$

\section{ВВЕДЕНИЕ}

В течение последних лет многочисленные научные работы были сфокусированы на исследованиях сферических гибридных двухслойных наночастиц из-за уникальных зависимостей их физических и химических свойств от размеров [1-5]. Плазмонные свойства наноразмерных двухслойных наночастиц зависят от их размеров, формы, оптических свойств материалов ядра и оболочки и окружаюшей среды [5-9]. Посредством изменения размеров ядра и толщины оболочки плазмонный резонанс гибридной двухслойной наночастицы может быть сдвинут в широком диапазоне оптического спектра.

Двухслойные наночастицы используются в медицине [10-13], катализе [14, 15], сенсорах [16], солнечной энергетике [17-20] и в других областях.

Естественное окисление металлических наночастиц происходит в окружающих жидких или газообразных средах [10-15, 17-20]. Это приводит к образованию достаточно тонкой оболочки из окисла металла с толшиной порядка 5-10 нм на поверхности металлической наночастицы. Для определенных целей физическими или химическими методами создают окисные оболочки для предохранения металлических наночастиц, размешаемых в химически активных средах. Следовательно, кроме исследования чистых металличе- ских наночастиц для реального применения необходимо исследовать оптические свойства металлических наночастиц с окисными оболочками [21-27]. Кроме того, в последнее время начинают активно использоваться однородные наночастицы из окислов металлов.

В связи с этим представляет значительный интерес исследование оптических свойств наночастиц при переходе от однородной металлической наночастицы к однородной наночастице из окисла данного металла через образование гибридной двухслойной наночастицы с ядром из металла и оболочкой из окисла данного металла. Это позволяет проанализировать изменение оптических свойств при образовании оболочки из окисла данного металла при помещении металлической наночастицы в окисляющую среду.

В данной работе исследовано влияние оптических характеристик, структур и размеров наночастиц на индикатрисы рассеянного излучения для нескольких длин волн. Знание индикатрис рассеяния излучения важно при обшем анализе влияния оптических характеристик наночастищ на последующие процессы, возникающие при взаимодействии интенсивного оптического излучения с наночастицей. Кроме того, важным является выяснение факта рассеяния излучения в переднюю (от $90^{\circ}$ до $270^{\circ}$ ) и заднюю (от $270^{\circ}$ до $90^{\circ}$ ) полусферы. Это связано со следуюшими обстоятельствами. 
Наночастица поглощает и рассеивает падающее на нее излучение. Поглощенное наночастицей излучение выбывает из последуюшего оптического рассмотрения, а рассеянное излучение распространяется в среде с наночастицами и участвует в последующем акте поглощения и рассеяния и т.д.

В наноэнергетике, солнечных ячейках и системах прямого оптического нагрева наночастиц [21-27] представляет интерес рассеяние излучения с индикатрисами, близкими к сферическим. В этом случае рассеянное излучение будет равномерно распределяться по всем направлениям от рассеивающей наночастицы и потенциально эффективно поглощаться и рассеиваться соседними наночастицами. В качестве маловероятного, но потенциально весьма интересного случая возможна ситуация, когда излучение будет максимально рассеиваться в диапазонах $120^{\circ}-60^{\circ}$, $240^{\circ}-300^{\circ}$. Это, возможно, приведет к уменьшению рассеяния в переднюю и заднюю полусферы и обеспечит эффективное поглошение излучения в системах прямого поглощения излучения с существенно большими геометрическими размерами в указанном диапазоне рассеяния излучения. В случае доминирующего рассеяния излучения наночастицей в переднюю или заднюю полусферы процессы рассеяния будут выводить излучение из облучаемой области наножидкости и резко снижать эффективность конечного поглощения излучения наночастицами и их нагревание.

В некоторых применениях представляет значительный интерес использование наночастиц в качестве агентов, препятствуюших распространению излучения вглубь среды, в которой они размешены. В этом случае рассеяние излучения наночастицами в заднюю полусферу должно доминировать над рассеянием излучения в переднюю полусферу. При этом должно быть существенным поглощение излучения наночастицами, что будет дополнительно способствовать ослаблению излучения.

Представляет также интерес использование наночастиц в качестве агентов, способствующих распространению излучения вглубь среды, в которой они размешены. Это может быть использовано в системах концентрации (фокусировки) потока излучения на некоторой поверхности, на которой располагается среда с такими наночастицами. В этом случае рассеяние излучения наночастицами в переднюю полусферу должно доминировать над рассеянием излучения в заднюю полусферу. Дополнительно наночастицы должны рассеивать энергию излучения много больше, чем ее поглошать.

Соответствующим подбором таких характеристик наночастиц, как их структура, размер, мате- риал, форма для конкретных длин волн могут быть достигнуты данные результаты.

\section{АНАЛИЗ УГЛОВЫХ ОПТИЧЕСКИХ ХАРАКТЕРИСТИК ИЗЛУЧЕНИЯ, РАССЕЯННОГО ДВУХСЛОЙНЫМИ НАНОЧАСТИЦАМИ СИСТЕМЫ МЕТАЛЛ-ОКИСЕЛ}

В настоящей работе на основе теории дифракции электромагнитного излучения на сферической многослойной частице [28] представлены результаты расчета индикатрис рассеяния излучения с длинами волн $\lambda=300,560$ и 1000 нм однородными наночастицами с радиусами $r_{0}=50,75$, 100 нм и двухслойными наночастицами с радиусами ядра $r_{0}=40,65,90$ нм и толщиной оболочки $r_{1}=10$ нм. В качестве материалов однородных наночастиц использованы металлы $\mathrm{Ti}, \mathrm{Ni}, \mathrm{Zn}$ и окислы этих металлов $\mathrm{TiO}_{2}, \mathrm{NiO}, \mathrm{ZnO}$. В качестве материалов ядра двухслойных наночастиц использованы металлы $\mathrm{Ti}, \mathrm{Ni}, \mathrm{Zn}$, в качестве оболочек - окислы этих металлов $\mathrm{TiO}_{2}, \mathrm{NiO}, \mathrm{ZnO}$ соответственно. Были выбраны следуюшие длины волн излучения: $\lambda=300$ нм (спектральная область ультрафиолетового излучения), 560 нм (видимый спектр, соответствуюший максимальной интенсивности в спектре солнечного излучения) и 1000 нм (инфракрасная область излучения). Следовательно, в данном случае рассматривается влияние не только самой длины волны излучения, но и принадлежность их к разным областям спектра оптического излучения. Металлы Ti, $\mathrm{Ni}$, $\mathrm{Zn}$ и окислы этих металлов $\mathrm{TiO}_{2}, \mathrm{NiO}, \mathrm{ZnO}$ широко используются для наночастиц в солнечной термической наноэнергетике [21-27] и для зашиты тканей человека от солнечного излучения.

Индикатрисы рассеяния показывают относительную интенсивность рассеянного наночастицей излучения по различным направлениям в диапазоне изменения углов рассеяния от $0^{\circ}$ до $360^{\circ}$. Отметим, что картины индикатрис рассеянного излучения являются симметричными относительно линии $180^{\circ}-0^{\circ}$.

На рис. 1 представлены индикатрисы рассеяния излучения с длинами волн $\lambda=300,560$ и 1000 нм наночастицами $\mathrm{Ti}, \mathrm{TiO}_{2}$ с радиусами $r_{0}=$ $=50,75,100$ нм и двухслойными наночастицами системы $\mathrm{Ti}+\mathrm{TiO}_{2}$ с радиусами ядра $r_{0}=40,65$, $90 \mathrm{Hм}$, толшиной оболочки $\Delta r_{1}=10$ нм и внешним радиусом $r_{1}=50,75,100$ нм. Таким образом, объемы однородной и двухслойной наночастиц равны между собой. Излучение распространяется слева направо (от $180^{\circ}$ к $0^{\circ}$ ). Для наночастиц Тi для $\lambda=300,560$ нм индикатрисы рассеяния излучения вытянуты преимушественно вперед по направлению распространения излучения. Рассеяние излучения в переднюю полусферу сушественно до- 
(a)

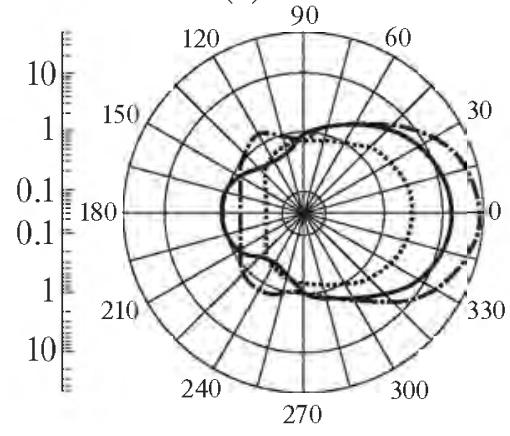

(г)

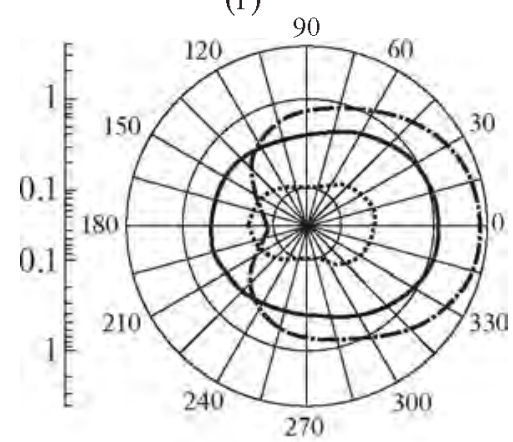

(ж)

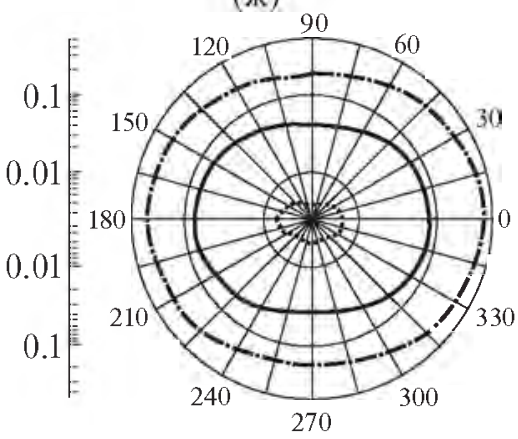

(б)

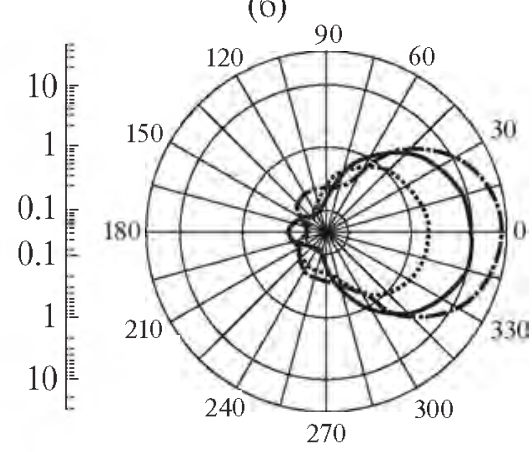

(д)

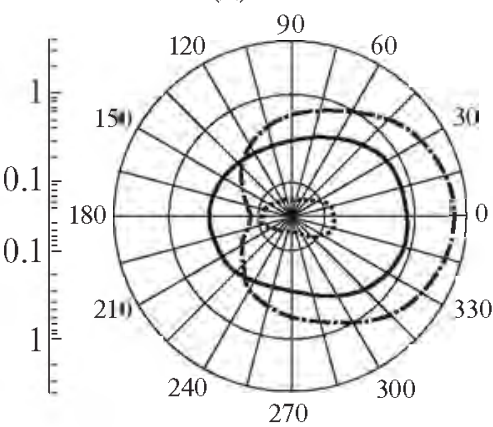

(3)

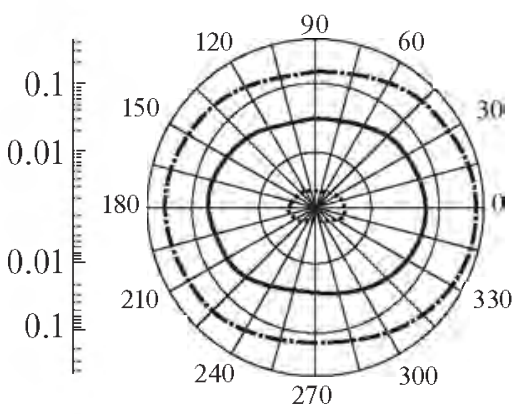

(в)

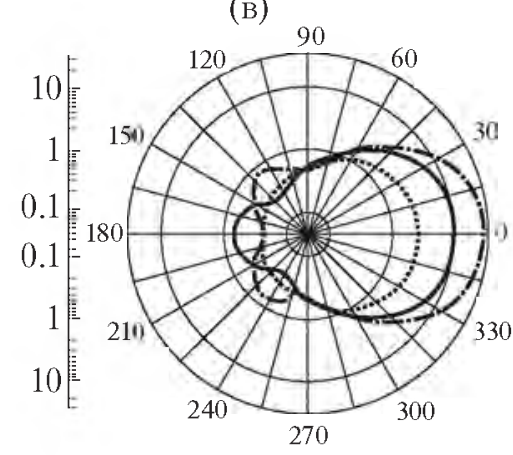

(e)

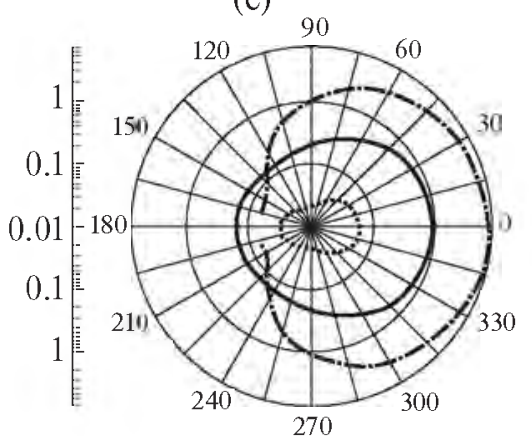

(и)

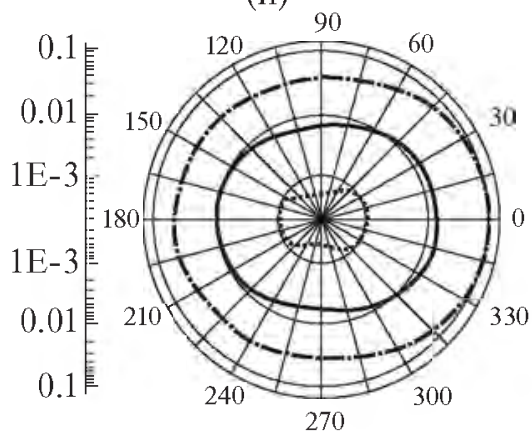

Pис. 1. Индикатрисы рассеяния излучения с длинами волн $\lambda=300$ (а, б, в), 560 (г, д, е) и 1000 нм (ж, 3, и) наночастицами $\mathrm{Ti}\left(\mathrm{a}\right.$, г, ж), $\mathrm{TiO}_{2}$ (в, е, и) с радиусами $r_{0}=50$ (точечная линия), 75 (сплошная линия), 100 нм (штрихпунктир) и наночастицами $\mathrm{Ti}+\mathrm{TiO}_{2}\left(\right.$ б, д, з) $\mathrm{c} r_{0}=40,65,90$ нм. $\Delta r_{1}=10$ нм. Излучение распространяется слева направо (от $180^{\circ} \mathrm{K} 0^{\circ}$ ).

минирует для $\lambda=300$ нм для представленных на рис. 1а и 1в однородных наночастиц $\mathrm{Ti}$ и $\mathrm{TiO}_{2}$ и особенно превалирует для двухслойных наночастиц $\mathrm{Ti}+\mathrm{TiO}_{2}$ (рис. 1б) практически для всех размеров. Возрастание длины волны излучения ведет к небольшому увеличению рассеяния в заднюю полусферу, особенно для $r_{0} \sim 50 \mathrm{Hм}$, а для $\lambda=$ $=1000$ нм (рис. 1ж, 13, 1и) приводит к практически симметричной картине индикатрис рассеяния для передней и задней полусфер.

С увеличением длины волны рассеянное излучение существенно падает, особенно для малых размеров наночастицы. Для $\lambda=300$ нм количество рассеянного излучения слабо зависит от раз- мера наночастицы. Однако для $\lambda=560$ нм и, особенно, для $\lambda=1000$ нм, доля рассеянного излучения резко увеличивается с ростом радиуса наночастицы. Возникновение окисной оболочки из $\mathrm{TiO}_{2}$ на металлическом Ті-ядре приводит к небольшому качественному и количественному изменению картин рассеяния излучения. Объем оболочки с толшиной $\Delta r_{1}=10$ нм составляет примерно $48.8,34.9,27.1 \%$ соответственно от объема всей наночастицы с радиусом ядра $r_{0}=40,65$, 90 нм. Это означает, что образование сравнительно большой по сравнению с ядром оболочки в результате естественного окисления Ті-наночастицы в окисляющей среде (воздух, вода и т.д.) не оказывает существенного влияния на оптические 
(a)

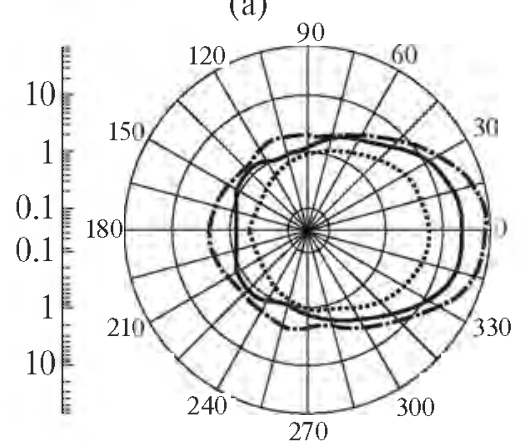

(г)

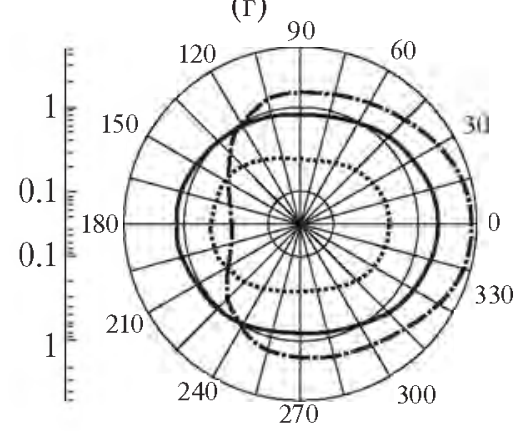

(ж) 90

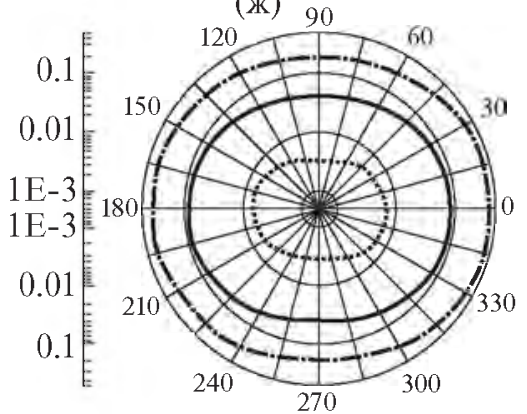

(б)

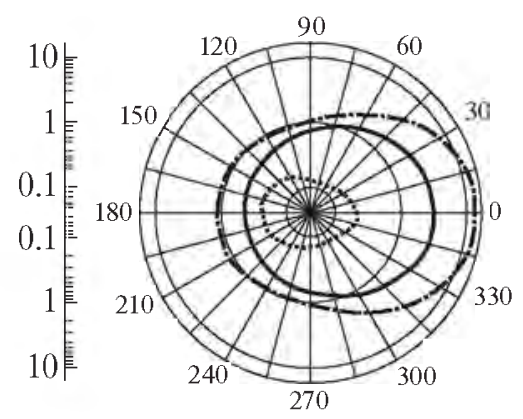

(д)

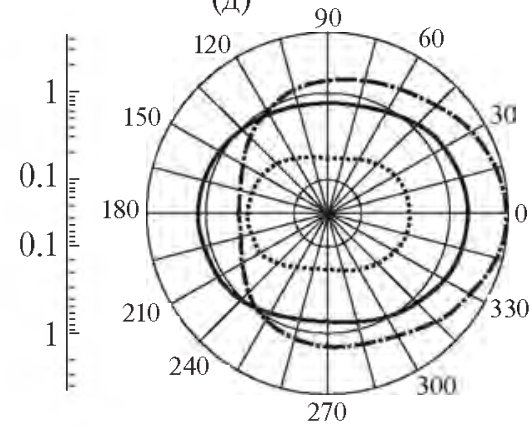

(3)

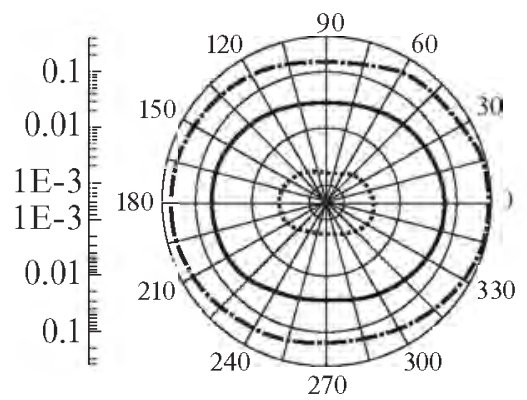

(в)

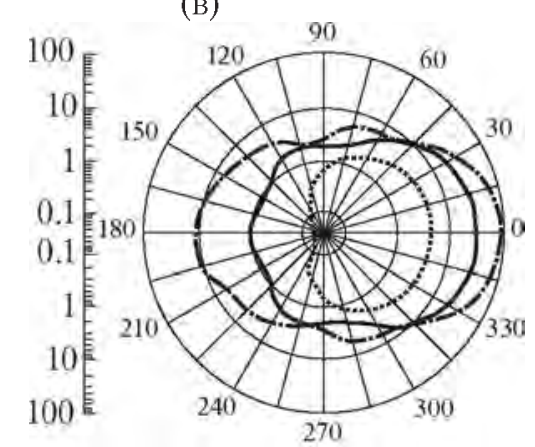

(e)

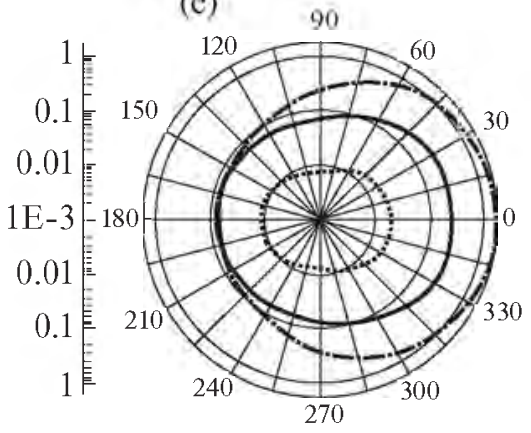

(и)

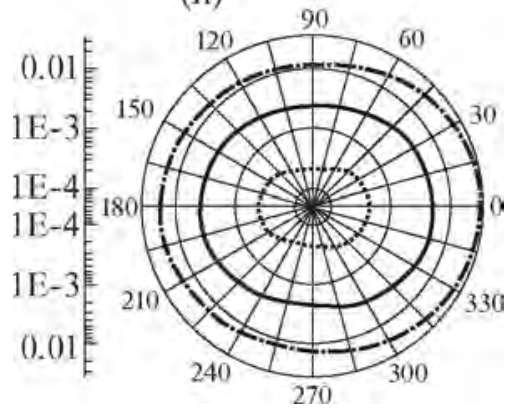

Рис. 2. Индикатрисы рассеяния излучения с длинами волн $\lambda=300$ (а, г, ж), 560 (б, д, з) и 1000 нм (в, е, и) наночастицами Zn (а, б, в), ZnO (г, д, е) с радиусами $r_{0}=50$ (точечная линия), 75 (сплошная линия), 100 нм (штрихпунктир) и наночастицами $\mathrm{Zn}+\mathrm{ZnO}$ (г, д, е) с $r_{0}=40,65,90$ нм. $\Delta r_{1}=10$ нм. Излучение распространяется слева направо (от $\left.180^{\circ} \mathrm{K} 0^{\circ}\right)$.

характеристики рассеяния излучения наночастицей, особенно для наночастиц с $r_{0}, r_{1}=100$ нм. Оптические характеристики рассеяния излучения окисной наночастицей $\mathrm{TiO}_{2}$ для $\lambda=300$ и 1000 нм незначительно качественно и количественно отличаются от рассеиваюших свойств наночастиц Тi. Однако для $\lambda=560$ нм различие в картинах рассеяния несколько больше.

На рис. 2 представлены индикатрисы рассеяния излучения с длинами волн $\lambda=300,560$ и 1000 нм наночастицами $\mathrm{Zn}, \mathrm{ZnO}$ с радиусами $r_{0}=$ $=50,75,100$ нм и $\mathrm{Zn}+\mathrm{ZnO}$ с $r_{0}=40,65,90 \mathrm{нм}$, $\Delta r_{1}=10$ нм. На длине волны $\lambda=300$ нм для однородной $\mathrm{Zn}$ наночастицы индикатриса рассеяния для $r_{0}=50$ нм вытянута больше вдоль направления распространения излучения, для двухслой- ной наночастицы $\mathrm{Zn}+\mathrm{ZnO}$ вытянутость индикатрисы рассеяния изменяется на обратную, а в случае однородной наночастицы из $\mathrm{ZnO}$ восстанавливается вытянутость вперед. На длине волны 560 нм индикатриса рассеяния для всех представленных наночастиц с ростом их радиуса вытягивается в направлении распространения излучения. В то же время на длине волны 1000 нм для всех представленных наночастиц и всех их радиусов форма индикатрисы рассеяния приближается к сферической, особенно для больших размеров наночастиц $\left(r_{0}>50 \mathrm{нм}\right)$.

На рис. 3 представлены индикатрисы рассеяния излучения с длинами волн $\lambda=300,560$ и 1000 нм наночастицами $\mathrm{Ni}, \mathrm{NiO}$ с радиусами $r_{0}=$ $=50,75,100$ нм и наночастицы $\mathrm{Ni}+\mathrm{NiO} \mathrm{c} r_{0}=40$, 
(a)

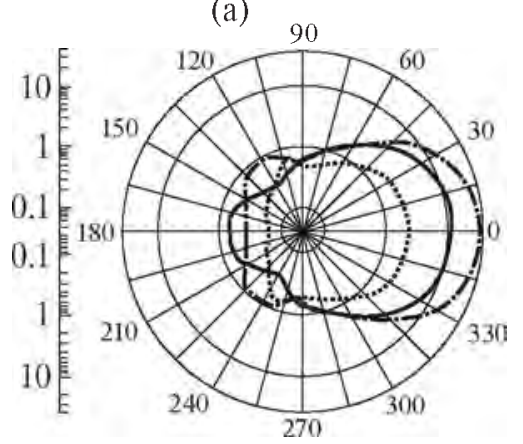

(г)

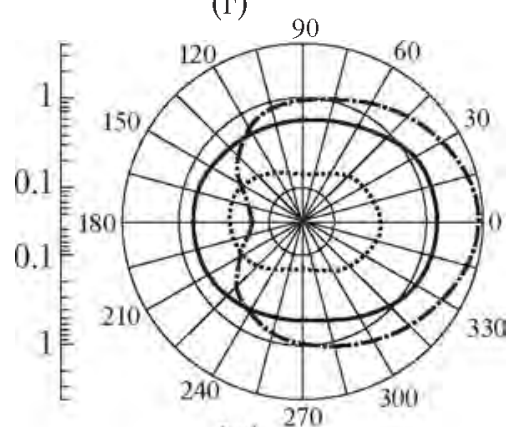

(ж)

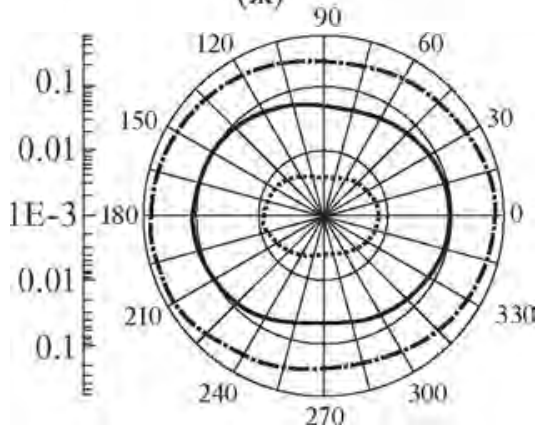

(б)
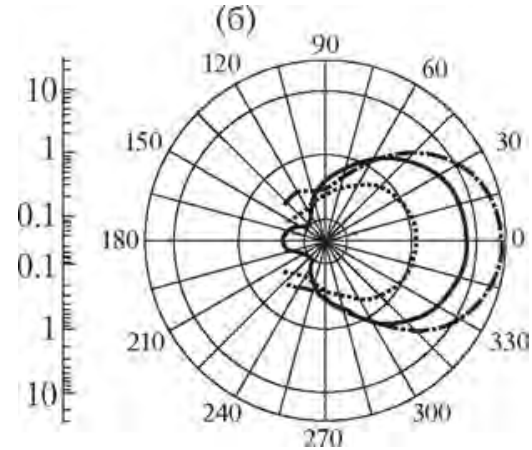

(Д)

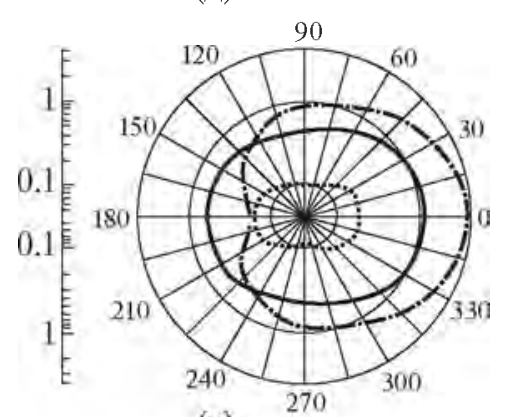

(3)

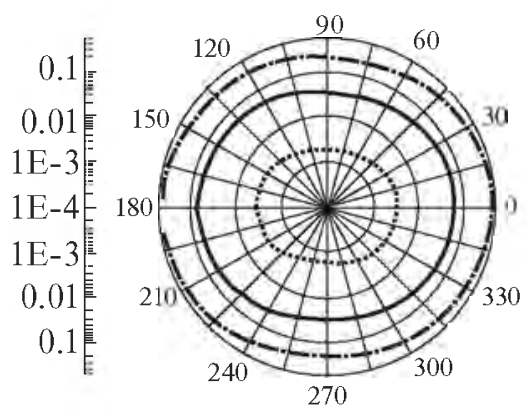

(B)

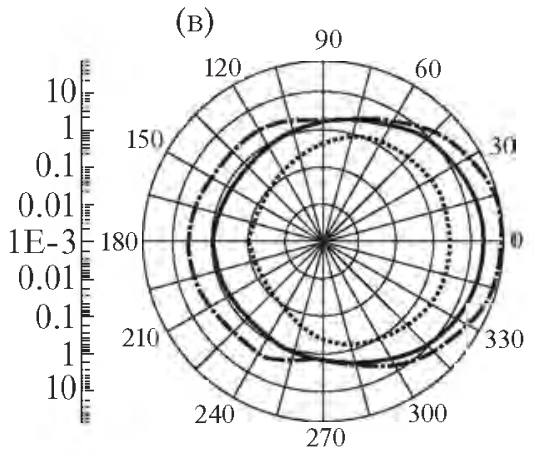

(e)

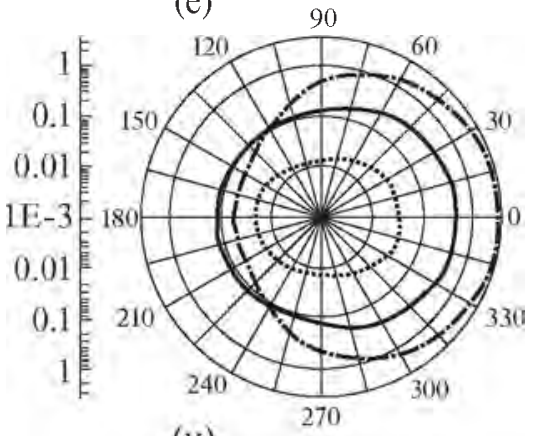

(1i)

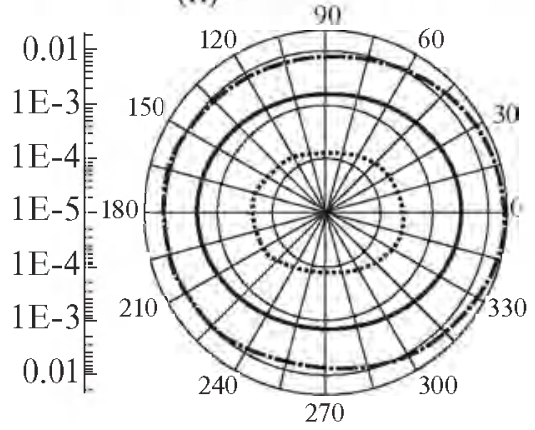

Рис. 3. Индикатрисы рассеяния излучения с длинами волн $\lambda=300$ (а, г, ж), 560 (б, д, з) и 1000 нм (в, е, и) наночастицами $\mathrm{Ni}(\mathrm{a}, \sigma, \mathrm{s}), \mathrm{NiO}$ (ж, 3, и) с радиусами $r_{0}=50$ (точечная линия), 75 (сплошная линия), 100 нм (штрихпунктир) и наночастицами $\mathrm{Ni}+\mathrm{NiO}$ (г, д, е) с $r_{0}=40$ (точечная линия), 65 (сплошная линия), 90 нм (штрихпунктир), $\Delta r_{1}=10$ нм.

Излучение распространяется слева направо (от $\left.180^{\circ} \mathrm{K} 0^{\circ}\right)$.

65, 90 нм и толщиной оболочки $\Delta r_{1}=10$ нм. На длине волны $\lambda=300$ нм индикатриса рассеяния для наночастиц $\mathrm{Ni}$ и $\mathrm{Ni}+\mathrm{NiO}$ вытягивается в направлении распространения излучения с ростом радиуса наночастицы. В то же время для наночастицы $\mathrm{NiO}$ индикатриса становится близка к сферической форме с ростом радиуса.

На длине волны 560 нм для всех наночастиц и всех их радиусов индикатриса рассеяния в большей или меньшей степени вытянута вдоль направления распространения излучения. В то же время на длине волны 1000 нм для всех представленных разновидностей наночастиц и всех их радиусов форма индикатрисы рассеяния близка к сферической.

\section{ЗАКЛЮЧЕНИЕ}

Проведен анализ рассеивающих свойств двухслойных гибридных наночастиц с целью их возможного применения в оптических и лазерных нанотехнологиях. В частности, особый интерес представляют оптические свойства при образовании окисной оболочки на металлическом ядре наночастицы при помещении в окисляюшую среду. Наличие окисной оболочки может быть использовано для предохранения наночастиц в некоторых химически активных средах и в некоторых других условиях.

Установлено влияние параметров наночастиц и характеристик излучения на формы индикатрисы рассеяния излучения на наночастище и на перераспределение излучения вокруг наночастицы 
по различным направлениям. Наличие окисной оболочки на металлическом ядре из титана и никеля по сравнению с чисто металлической или чисто окисной диэлектрической наночастицей приводит к сушественному уменьшению обратного рассеяния для $\lambda=300$ нм.

Для ультрафиолетовой длины волны 300 нм и длины волны видимого света 560 нм увеличение радиусов однородных и двухслойной наночастиц в основном приводит к увеличению рассеяния света вдоль направления распространения падающего излучения. На длине волны 1000 нм инфракрасного излучения форма индикатрисы рассеянного света для всех представленных наночастиц и для всех их размеров близка к сферической.

\section{СПИСОК ЛИТЕРАТУРЫ}

1. Steinbrück A., Csaki A., Ritter K., Leich M., Kohler J., Fritzsche W. // J. Nanopart. Res. 2009. V. 11. № 3. P. 623.

2. Major K., De C., Obare O. // Plasmonics. 2009. V. 4. № 1. P. 61.

3. Guerro-Martinez A., Perez-Juste J., Liz-Marzan L. // Adv. Mater. 2010. V. 22. № 11. P. 1182.

4. Chaudhuri R., Paria S. // Chem. Rev. 2012. V. 112. № 4. P. 2373.

5. Cortie M.B., McDonagh A.M. // Chem. Rev. 2011. V. 111. № 6. P. 3713.

6. Bohren C.F., Huffman D.R. Absorption and Scattering of Light by Small Particles. New York: Wiley, 1983. $664 \mathrm{p}$.

7. Shaviv E., Schubert O., Alves-Santos M., Goldoni G., Di Felice R., Vallee F., Del Fatti N., Sonnichsen C. // ACS Nano. 2011. V. 5. № 6. P. 4712.

8. Pustovalov V.K., Astafyeva L.G., Fritzsche W. // Plasmonics. 2012. V. 7. № 3. P. 469.

9. Laaksonen K., Suomela S., Puisto S.R., Rostedt N.K.J., Ala-Nissila T., Nieminen R.M. // JOSA. B. 2013. V. 30. № 2. P. 338 .
10. Hirsch L.R., Gobin A.M., Tam F, Drezek R.A., Halas N.J., West J.L. // Ann. Biomed. Eng. 2006. V. 34. № 1. P. 15.

11. Pustovalov V., Astafyeva L., Jean B. // Nanotechnology. 2009. V. 20. № 22. P. 225105.

12. Cheng F. Y., Chen C.-T., Yeh C.-S. // Nanotechnology. 2009. V. 20. № 42. P. 425104.

13. Wang J., Byrne J.D., Napier M.E., De Simone J.M. // Small. 2011. V. 7. № 14. P. 1919.

14. Narayanan R., El-Sayed M.A. // Top. Catal. 2008. V. 47. № 1. P. 15-23.

15. Adleman J.R., Boyd D.A., Goodwin D.G., Psaltis D. // Nano Lett. 2009. V. 9. № 12. P. 4417.

16. Steinbrück A., Stranik O., Csaki A., Fritzsche W. // Analyt. Bioanalyt. Chem. 2011. V. 401. № 4. P. 1241.

17. Catchpole K.R., Polman A. // Opt. Express. 2008. V. 16. № 26. P. 21793.

18. Radousky H.B., Liang H.E. // Nanotechnology. 2012. V. 23. P. 502001 (35 pp.).

19. Mlinar V. // Nanotechnology. 2013. V. 24. P. 042001 (11 pp).

20. Erickson D., Sinton D., Psaltis D. // Nature Photonics. 2011. V. 5. № 4. P. 583.

21. Valverde-Aguillar G., Garcia-Macedo J.A., RenteriaTapia V. // J. Nano Res. 2008. V. 3. № 1. P. 103.

22. Santillan J.M.J., Scaffardi L.B., Schinca D.C.//J. Phys. D. 2011 . V. 44. № 10. P. 105104.

23. Yang Z., He L., Chen J., Cong H., Ye H. // J. Mater. Res. 2003. V. 18. №. 2. P. 272.

24. Peng Y.-J., Zhang S.-P., Wang Y.-H., Yang Y.-Q.//Chinese Physics B. 2008. V. 17. № 9. P. 3505.

25. Martin J.E., Herzing A.A., Yan W., Li X.-Q., Koel B.E., Kiely C.J., Zhang W.-X. // Langmuir. 2008. V. 24. № 8. P. 4329.

26. Zeng H., Li Z., Cai W., Cao B., Liu P., Yang S. //J. Phys. Chem. B. 2007. V. 111. № 51. P. 14311.

27. Ghodselahi T., Vesaghi M.A., Shafiekhani A. // J. Phys. D. 2009. V. 42. № 1. P. 015308.

28. Babenko V.A., Astafyeva L.G., Kuzmin V.N. Electromagnetic Scattering in Disperse Media. Inhomogeneous and Anisotropic Particles. Chichester, UK: Praxis Publishing Ltd., 2003. 434 p. 